Session 1726

\title{
Undergraduate Heat Transfer Experiment: Measurement of Thermal Conductivity of Liquids and Gases
}

\author{
Hosni I. Abu-Mulaweh, Donald W. Mueller, Jr. \\ Department of Engineering \\ Indiana University-Purdue University Fort Wayne \\ Fort Wayne, IN 46805, USA
}

\begin{abstract}
Determining physical properties of substances is an important subject in many advanced engineering applications. Physical properties of liquids and gases, such as thermal conductivity, play an important role in the design of a wide variety of engineering applications such as heat exchangers. This paper describes an undergraduate junior-level heat transfer experiment designed for students to determine the thermal conductivity of liquids and gases. Details of the experimental apparatus, testing procedure, data reduction, and sample results are presented. One of the objectives of this experiment is to strengthen and reinforce some of the heat transfer concepts, such as conduction, covered in the classroom lectures. The experimental setup is simple, the procedure is straightforward, and students' feedback is very positive.
\end{abstract}

\section{Introduction}

Heat transfer is a basic and very important topic that deals with energy and has long been an essential part of mechanical engineering curricula all over the world. Heat transfer processes are encountered in a large number of engineering applications. It is essential for thermal engineers to understand the principles of thermodynamics and heat transfer and be able to employ the rate equations that govern the amount of energy being transferred. However, the majority of students perceive these topics as difficult.

To make the subject of heat transfer a more pleasant experience for the undergraduate mechanical engineering students at Indiana University-Purdue University Fort Wayne, the students are required to take a junior-level heat transfer laboratory. The different experiments in this laboratory enhance and add another dimension to the teaching/learning process of the subject of heat transfer. One of the objectives of this heat transfer laboratory is to familiarize the students with different experimental methods, techniques, and devices that can be employed to study heat transfer problems. One important experiment in this laboratory, which is the subject of this paper, is the measurement of the thermal conductivity of liquids and gases.

Physical properties of liquids and gases, such as thermal conductivity, play an important role in 
the design of a wide variety of engineering applications such as heat exchangers. Convective heat transfer coefficients in these devices are usually computed using correlations that require thermal conductivity data of the working fluid (liquid or gas).

A review of the literature indicates that there are several studies that report different techniques to measure the thermal conductivity of liquids and gases. Kaye and Higgins [1] suggested a guarded hot-plate method for measuring thermal conductivity of liquids. Keyes and Sandell [2] and Vines [3] used a concentric-cylinder arrangement for the measurement of thermal conductivity of gases. This paper presents a technique/method that can be employed for the measurement of the thermal conductivity of both liquids and gases.

\section{Theory}

Thermal conduction is a very important and a major topic in the study of heat transfer [4, 5]. Conduction is the transfer of energy from energetic particles of a substance to the adjacent less energetic ones as a result of interactions between the particles. Conduction can take place in solids, liquids, or gases. In gases and liquids, conduction is due to the collision and diffusion of the molecules during their random motion. The rate of heat conduction is proportional to the area and the temperature difference, and inversely proportional to the thickness of the material. The constant of proportionality is the thermal conductivity.

Thus the thermal conductivity, $\mathrm{k}$, of a material is defined as the rate of heat transfer through a unit thickness of the material per unit area per unit temperature difference. Therefore it is a measure of how fast heat will flow in the material. A large value for thermal conductivity indicates that the material is a good conductor, and a low value indicates that the material is a poor conductor or a good insulator.

The thermal conductivities of materials vary with temperature. This variation, for some materials over certain temperature ranges, is small enough to be neglected; but for many cases, such as liquids and gases, the variation of the thermal conductivity with temperature is significant.

\section{Experimental Apparatus}

The experimental apparatus, shown schematically in Fig. 1, is a compact unit made by P.A. Hilton Ltd. It consists of two parts: Test Module and Control Panel.

Test Module: It is a plug and jacket assembly that consists of a cylindrical heated plug and cylindrical water cooled jacket. The fluid (liquid or gas) whose thermal conductivity is to be measured fill a small radial clearance between the heated plug and the water cooled jacket. It should be noted that the clearance is made small in size to prevent natural convection in the fluid. 


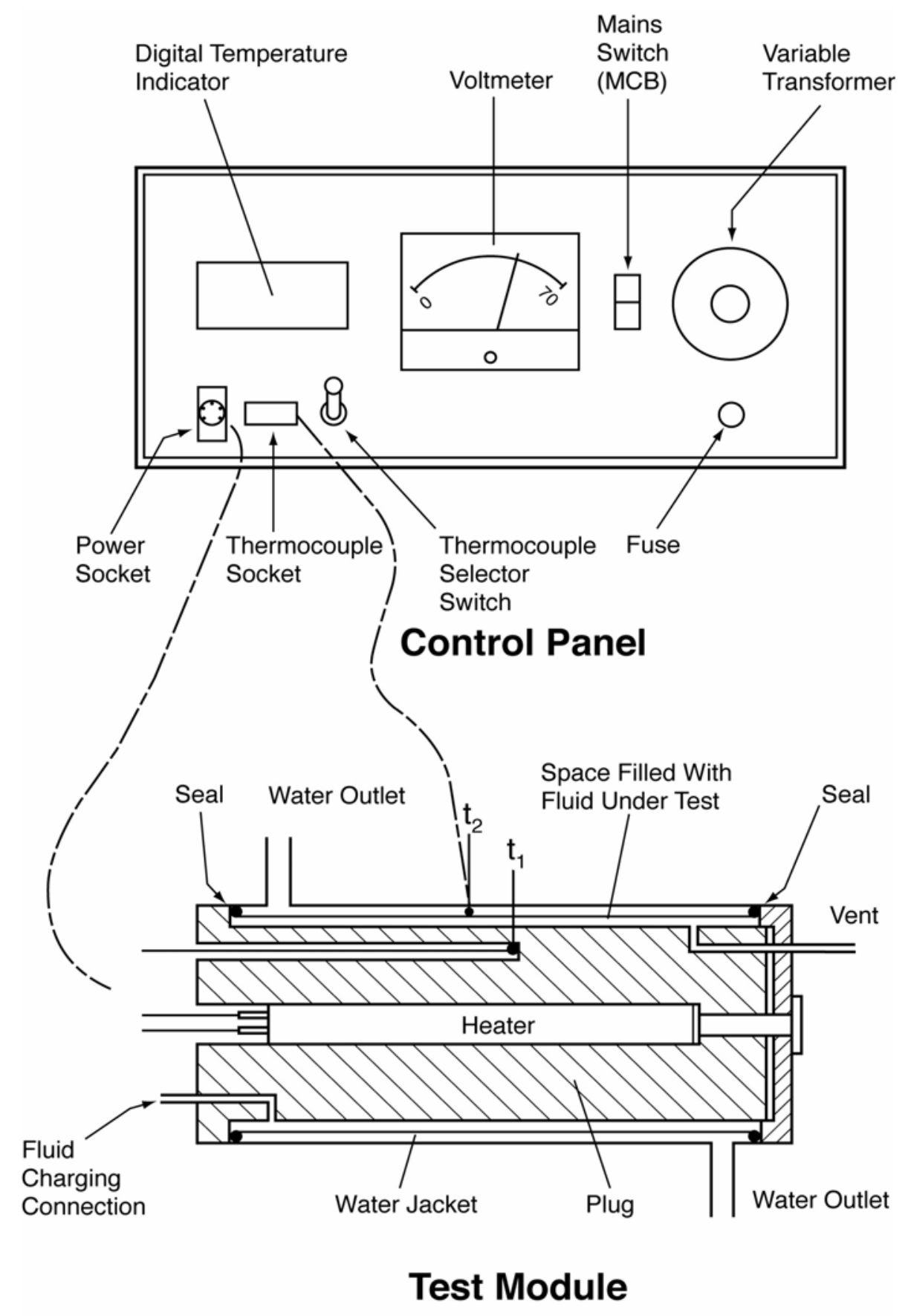

Figure 1: Schematics of the experimental apparatus and instrumentation 
The cylindrical plug is made of aluminum (to reduce thermal inertia and temperature variation) with a built-in cylindrical heating element and temperature sensor (thermocouple). The temperature sensor is inserted into the plug close to its external surface. The plug also has ports for the introduction and venting of the fluid (liquid or gas) whose thermal conductivity is to be measured. The plug is placed in the middle of the cylindrical water jacket. The water jacket is constructed from brass and has a water inlet and drain connections. A thermocouple is also fitted to the inner sleeve of the water jacket.

Control Panel: The test module is connected to the control panel (a small console) by flexible cables for the voltage supplied to the heating element. The control panel includes all necessary electrical wiring with variable transformer, power transducer, temperature controller/indicator, digital displays for temperature, analogue meter for voltage, and thermocouple selector switch.

IV. Determination of the Incidental Heat Transfer (or Calibration)

Before using the unit to measure a thermal conductivity of a fluid (liquid or gas), the unit must be calibrated. This is because not all power input is transferred by conduction through the test fluid, some energy (incidental heat transfer) will be lost to the surroundings and some will be radiated across the annulus. In this calibration process, the students will generate a curve that characterizes this incidental heat loss. The incidental heat transfers in the unit are determined by using air (whose thermal conductivity is well known and documented) in the radial space.

Procedure: The following is a brief summary of the procedure to carry out the calibration of the unit (Refer to Fig. 2)

1. Set up the equipment and make the necessary connections.

2. $\quad$ Pass water through the jacket at about 3 liters per min.

3. Connect the small flexible tubes to the charging and vent unions.

4. Close off the tubing with a pure air sample trapped in the device.

5. Switch on the electrical supply.

6. $\quad$ Adjust the variable transformer to give about $10 \mathrm{~V}$.

7. At intervals check the temperature of the plug, $T_{1}$, and jacket, $T_{2}$, and when they are stable, record their values and also the voltage.

8. Repeat steps 6 and 7 for $20 \mathrm{~V}, 30 \mathrm{~V}, 40 \mathrm{~V}, 50 \mathrm{~V}$, and $60 \mathrm{~V}$. 


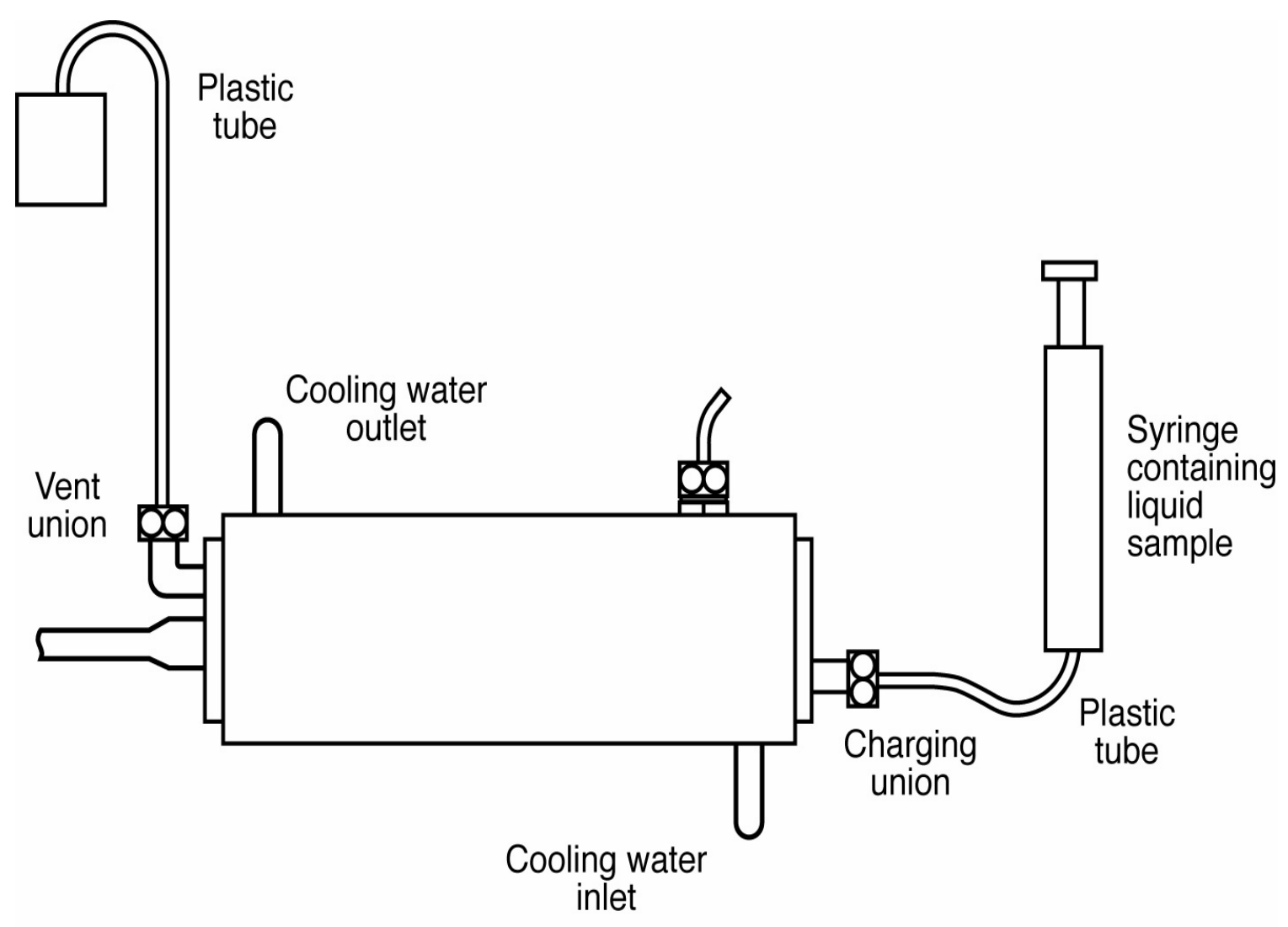

Figure 2: Schematic of experimental apparatus

\section{Calculations:}

1. Find the thermal conductivity of the air, $\mathrm{k}_{\mathrm{air}}$, at the average temperature, $\mathrm{T}_{\mathrm{ave}}=$ $\left(T_{1}+T_{2}\right) / 2$. Temperature dependent thermal conductivity values for air are found in any heat transfer textbook such as Incropera and DeWitt [4] and Özisik [5].

2. Calculate the rate of heat conducted through the air lamina, $Q_{c}$, from Fourier's Law, i.e.,

$$
Q_{c}=k A \frac{\Delta T}{\Delta r}
$$

where the area is $A=0.0133 \mathrm{~m}^{2}$, the radial clearance is $\Delta r=0.34 \mathrm{~mm}$, and the temperature difference is $\Delta T=T_{1}-T_{2}$.

3. Calculate the rate of electrical heat input, $Q_{e}$, from 


$$
Q_{e}=\frac{V^{2}}{R},
$$

where $V$ is the voltage and $R$ is the resistance of element, $R=54.8 \Omega$.

4. Find the incidental heat transfer, $Q_{i}$, (loss, radiation, etc.). The incidental heat transfer is the difference between the electrical heat input and the heat conducted through the fluid in the radial clearance, i.e.,

$$
Q_{i}=Q_{e}-Q_{c}
$$

\section{$\underline{\text { Results: }}$}

From the measured data and the results obtained above, a calibration curve of the incidental heat transfer, $Q_{i}$, against the average temperature, $\mathrm{T}_{\text {ave, }}$, can be generated. Figure 3 presents the calibration curve of the unit that was obtained by one of the laboratory student groups. As can be seen from the figure, the incidental heat transfer increases linearly as the average temperature increases.

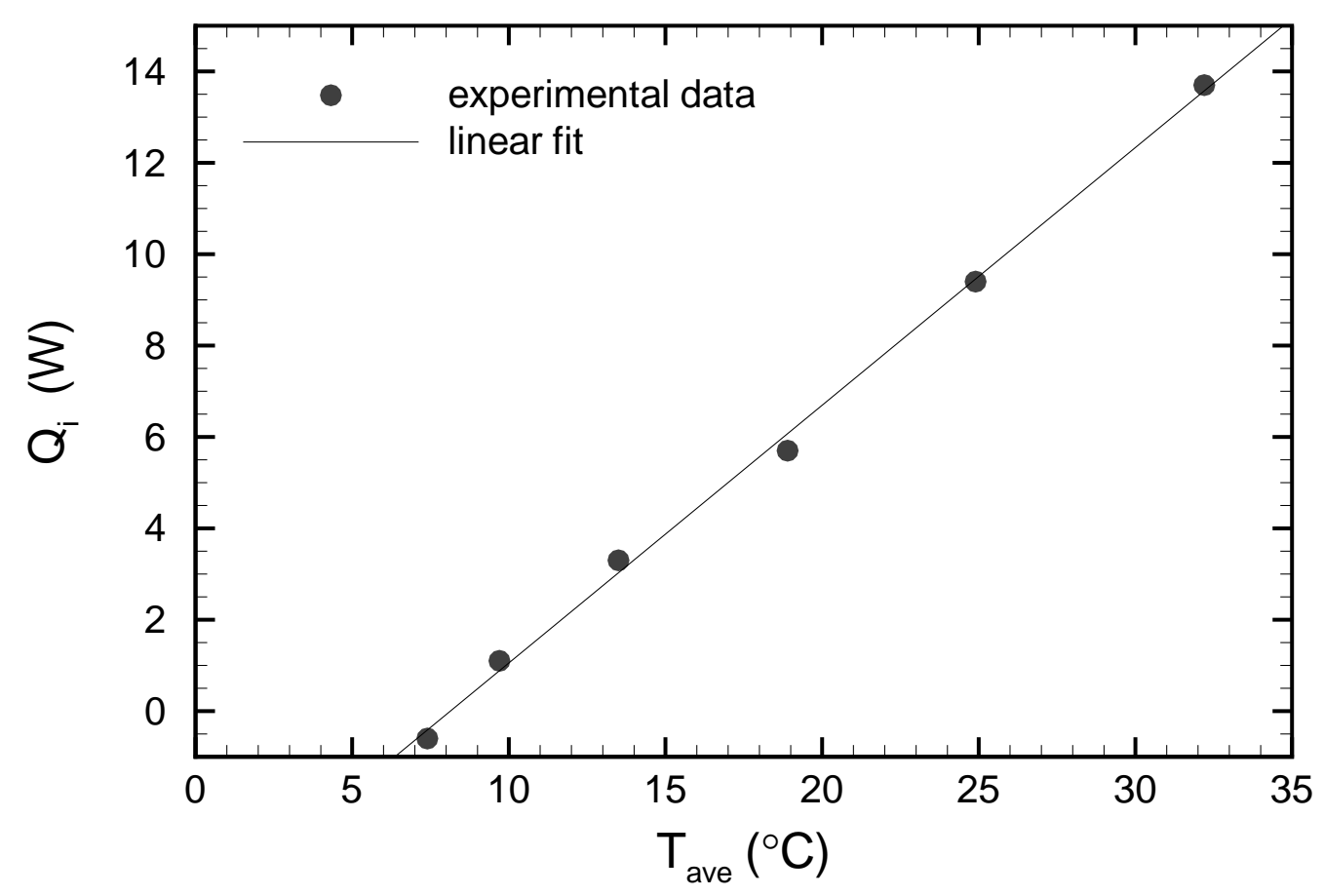

Figure 3: Calibration curve 


\section{Determination of the Thermal Conductivity of a Liquid or Gas}

Once the calibration curve is obtained and the unit is cleaned and reassembled, the students can then introduce the fluid (liquid or gas) to be tested into the radial clearance. It should be noted that it is important to ensure that no bubbles exist if the test fluid is liquid. Water is then passed through the jacket and the variable transformer is adjusted to the desired voltage. The voltage value is chosen to give a reasonable temperature difference and heat transfer rate. When stable, the plug and jacket temperatures and the voltage are recorded.

The incidental heat transfer rate, $Q_{i}$, is then found from Fig. 3 at the average temperature, $T_{\text {ave }}$. Once the rate of the incidental heat transfer is determined, the rate of heat conducted through the test fluid (liquid or gas) is then found from Equation (3) as $Q_{c}=Q_{e}-Q_{i}$, and the thermal conductivity of the test fluid (liquid/gas) can be calculated from Equation (1), i.e. $k=Q_{c} \Delta r /(A \Delta T)$. It is recommended that this procedure is repeated at another voltage value to ensure the consistency of the measurements.

In addition, an uncertainty analysis of the measured thermal conductivity of the test fluid is performed. The calibration process can be used to estimate the uncertainty in the heat conduction across the sample. The conduction across the sample is found from Fourier's Law which, for this situation, is given in Eq. 1 as

$$
Q_{c}=k A \frac{\Delta T}{\Delta r}=k A \frac{\left(T_{1}-T_{2}\right)}{\Delta r} .
$$

Application of the standard uncertainty procedure as described in Ref. [6] to Eq. (4) yields

$$
\left(\frac{U_{Q_{c}}}{Q_{c}}\right)^{2}=2\left[\left(\frac{U_{k}}{k}\right)^{2}+\left(\frac{U_{\Delta r}}{\Delta r}\right)^{2}+\left(\frac{U_{A}}{A}\right)^{2}+\left(\frac{U_{T}}{T}\right)^{2}\right],
$$

where $U$ represents the uncertainty in the quantity indicated with the subscript and uncertainty in the temperature measurements are assumed equal, i.e., $U_{T_{1}} \approx U_{T_{2}} \approx U_{T}$. The radial clearance and the area are provided by the manufacturer and assumed to be very accurate so that $U_{\Delta r} \approx U_{A} \approx 0$. The thermal conductivity of the air at a given temperature is assumed to be known within $2.5 \%$, and the uncertainties in the temperature measurements are assumed to be less than $1^{\circ} \mathrm{C}$. Thus, the uncertainty in the heat conduction across the sample is estimated to be less than $4 \%$.

The uncertainty in the thermal conductivity can be found with a similar procedure. First, Eq. (4) is rearranged to solve for the thermal conductivity, i.e. 


$$
k=\frac{Q_{c} \Delta r}{A \Delta T}=\frac{Q_{c} \Delta r}{A\left(T_{1}-T_{2}\right)}
$$

and the procedure outlined in Ref. [6] yields

$$
\left(\frac{U_{k}}{k}\right)^{2}=\left(\frac{U_{Q_{c}}}{Q_{c}}\right)^{2}+\left(\frac{U_{\Delta r}}{\Delta r}\right)^{2}+\left(\frac{U_{A}}{A}\right)^{2}+\left[\frac{T_{1}}{T_{1}-T_{2}}\right]^{2}\left(\frac{U_{T_{1}}}{T_{1}}\right)^{2}+\left[\frac{T_{2}}{T_{1}-T_{2}}\right]^{2}\left(\frac{U_{T_{2}}}{T_{2}}\right)^{2} .
$$

As previously, the radial dimension and the area are assumed to be very accurate so that $U_{\Delta r} \approx U_{A} \approx 0$, and the uncertainty in the temperature measurements are assumed to be equal, $U_{T_{1}} \approx U_{T_{2}} \approx U_{T}$, so that Eq. (7) can be simplified to

$$
\left(\frac{U_{k}}{k}\right)^{2}=\left(\frac{U_{Q_{c}}}{Q_{c}}\right)^{2}+2\left(\frac{U_{T}}{T_{1}-T_{2}}\right)^{2}
$$

The uncertainty heat conduction across the sample was estimated be $4 \%$ and uncertainty in the temperature measurement was conservatively estimated to be $1^{\circ} \mathrm{C}$, so that the relative uncertainty in the thermal conductivity is less than $5 \%$ for typical experimental conditions.

The uncertainty in the measured results are estimated (at the 95\% confidence level) according to the procedure outlined by Moffat [6].

\section{Conclusion}

A heat transfer laboratory experiment in which undergraduate mechanical engineering students measure the thermal conductivity of a liquid or a gas is presented. In this experiment, students perform the calibration of the experimental apparatus and then employ the apparatus to determine the thermal conductivity of a liquid or a gas. This kind of experience serves to enhance the understanding of the transfer of thermal energy by undergraduate mechanical engineering students, while also exposing them to several important concepts involved in heat transfer.

\section{Bibliography}

1. Kaye, J.P.l and Higgins, W.F. (1928), “The Thermal Conductivities of Certain Liquids,” Proceedings of Royal SocietyLondon, Vol. 117, No. 459.

2. Keyes, F.G. and Sandell (1950), “New Measurements of Heat Conductivity of Steam and Nitrogen,” Trans. ASME, Vol. 72, p. 768.

3. Vines, R.G. (1960), "Measurements of Thermal Conductivities of Gases at High Temperatures,” Trans. ASME, Vol. 82C, P. 48.

4. Incropera, F.P and DeWitt, D.P. (2002), “Fundamentals of Heat and Mass Transfer,” John Wiley \& Sons, New York. 
5. Özisik, M.N. (1985), “Heat Transfer,” McGraw-Hill, New York.

6. Moffat, R.J. (1988), "Describing the Uncertainties in Experimental Results," Experimental Thermal and Fluids Science, Vol. 1, No. 1, p. 3.

HOSNI I. ABU-MULAWEH

Hosni I. Abu-Mulaweh is Professor of Mechanical Engineering at Indiana University-Purdue University, Fort Wayne, Indiana. He earned his B.S., M.S., and Ph.D. in Mechanical Engineering from the University of MissouriRolla, Rolla, Missouri. His areas of interest are Heat Transfer, Thermodynamics, and Fluid Mechanics.

DONALD W. MUELLER

Donald Mueller is an Assistant Professor of Mechanical Engineering at Indiana University-Purdue University, Fort Wayne, Indiana. He earned his B.S., M.S., and Ph.D. in Mechanical Engineering from the University of MissouriRolla, Rolla, Missouri. His areas of interest are Thermal-Fluid Sciences and Numerical Methods. 